\title{
¿Cuántos días se debe medir la dieta? \\ Variabilidad de la ingesta dietaria en Colombia
}

\author{
Woy many days must be measured diet? \\ Variability of dietary intake in Colombia
}

\begin{abstract}
During 2009-2011, information on dietary intake of 822 subjects between 18-59 years of age were collected. Each subject answered two 24-hours dietary recall $(\mathrm{R} 24 \mathrm{H})$ with a minimum interval of one week to avoid responses based on memory. In total, 1616 R24H were analyzed. The aim of this study was to describe the characteristics of the variability of twenty nutritional variables $(\mathrm{Vn})$, establish the sources and their contribution to the variability of the $\mathrm{V} n$, determine the number of days required to approach accurately the true average $V n$ and finally, to establish the feasibility study of the relationship between diet-health events disease in Colombia. The ratio of variances (Sw2/Sb2) was consistently higher in rural areas, except for energy and sodium. Calcium in rural areas and copper in urban areas had the lowest ratio Sw2/Sb2, 0.36 and 0.12 respectively. Vitamin $A$ in rural areas and sodium in urban areas, showed the largest ratio, 0.98 and 0.91 respectively. Socioeconomic status and geographic area are the main determinants of variability. For energy intake sex is the main source of variation. For macronutrients five repeated measurements are necessary to obtain the true average intake of these, with levels of error between $15 \%$ and $40 \%$. With the exception of vitamin A, all Sw2/Sb2 reasons are within values $<1$, which would still allow the development of nutritional epidemiology in Colombia.

Key words. Diet; epidemiologic methods; analysis of variance; sample size; Colombia.
\end{abstract}

\section{INTRODUCCIÓN}

En Colombia la prevalencia de anemia ferropénica esta entre $3 \%$ y $10 \%$, la de exceso de peso es $51,2 \%$, con evidencia de que el desplazamiento de la distribución aún no termina (1, 2). La proporción de muertes por eventos cardiovasculares en 1998 fue 21,6\% y en 2011 25,4\% (3). En 2011 los mediadores de la enfermedad cardiovascular como la hipertensión arterial y la diabetes alcanzaron en adultos prevalencias de $8,8 \%$ y $3,5 \%$ respectivamente, los eventos cardiovasculares son la primera causa de muerte en población general (4). La segunda es el cáncer, en donde a la dieta se le ha atribuido hasta 35\% de la causalidad $(4,5)$. La dieta es un factor ambiental que está en la vía causal de éstos y otros eventos negativos de salud (6-8). La principal dificultad para establecer relaciones entre dietaeventos de salud enfermedad, radica en la dificultad de contar con datos poblacionales del consumo dietario aspecto que en la región ha mejorado en la última década con el desarrollo de instrumentos de medición y de encuestas nacionales (1, 9-14). Complementariamente, en la región y Colombia se han venido experimentando cambios en los patrones alimentarios que pueden ser considerados expresiones no sólo de una transición nutricional sino alimentaria (15-20).
Oscar Fernando Herrán F. (1,2)

Sara Del Castillo M. (3)

Zulma Yanira Fonseca C. (3)

(1) Observatorio Epidemiológico de Enfermedades Cardiovasculares. Centro de Investigaciones Epidemiológicas (CIE). Universidad Industrial de Santander, Bucaramanga, Colombia

(2) Escuela de Nutrición y Dietética. Universidad Industrial de Santander. Colombia.

(3) Departamento de Nutrición Humana Observatorio de Seguridad Alimentaria y Nutricional (OBSAN) de la Universidad Nacional de Colombia. Colombia.

Dirigir la correspondencia a: Profesor

Oscar Fernando Herrán Falla Centro de Investigaciones Epidemiológicas (CIE) Facultad de Salud, Universidad Industrial de Santander Carrera 32 No. 29-31, tercer piso, oficina 304, Bucaramanga, Colombia Telefax: (57-7) 6345781

E-mail: herran@uis.edu.co / oscar.herran@gmail.com

Este trabajo fue recibido el 26 de Mayo de 2015 y aceptado para ser publicado el 25 de Julio de 2015.

La variabilidad estadística en el consumo dietario es un aspecto crucial a la hora de establecer la viabilidad en el estudio de relaciones entre dieta-eventos de salud enfermedad. Para lo anterior es necesario contar con dos a más mediciones de la dieta de un sujeto, con éstas se establece la variabilidad intra y entre individuos $(7,21-25)$. La información sobre la variabilidad de la dieta en cada contexto y estadio de la transición alimentaria, determina entre otros aspectos el número de días en que un sujeto debe encuestarse para estudiar las relaciones entre el consumo dietario de largo plazo y la enfermedad crónica o de sus mediadores como el exceso de peso, la hipertensión arterial, la diabetes y otros eventos $(7,21-27)$. Las características de la variabilidad también son útiles para estimar tamaños muestrales, corregir las medidas de efecto calculadas en estudios epidemiológicos y en general, para establecer el potencial de la epidemiología nutricional en cada sociedad (7, 21-27).

Los objetivos de este estudio fueron describir la variabilidad intra y entre sujeto en veintisiete variables nutricionales, las fuentes relativas que aportan a la variabilidad en éstas y con base en lo anterior, determinar el número de días necesarios para acercarse con precisión al verdadero promedio de las variables nutricionales en Colombia. 


\section{Sujetos y métodos}

El estudio se clasifica como analítico a partir de datos repetidos sobre la ingesta dietaria, y transversales del estado de nutrición y las condiciones socioeconómicas y ambientales de los individuos. Se desarrolló durante 2015 con datos recolectados entre 2009-2011 en cuatro ciudades de Colombia, Suramérica.

Bogotá es la capital de Colombia, en ella habitan alrededor de 9 millones de personas, Tenjo al igual que Sibaté son municipios urbano-rurales ubicados en el Área Metropolitana del Distrito Especial de Bogotá, cuentan con 20000 y 32000 habitantes respectivamente y sus economías, dependen de la agricultura, la ganadería, la prestación de servicios y la industria. Bucaramanga y su área Metropolitana cuenta con un millón de habitantes, su economía está ligada a la prestación de servicios.

Población estudiada. Durante 2009-2011, dentro del estudio diseñado y conducido por los autores de este informe "calidad de la dieta, percepción del hambre y seguridad alimentaria en población urbana y rural: estudio multicéntrico de evaluación de tecnología diagnóstica en Cundinamarca, Bogotá, Bucaramanga y su Area Metropolitana" (CDPH), se recolectó información sociodemográfica y dietaria en una muestra de 432 hogares y 1505 sujetos de los cuales 822 estaban en el rango de edad de 18-59 años. La información recolectada sobre consumo dietario en el CDPH se realizó con recordatorios repetidos de la ingesta de las últimas 24 horas (R24H), la información sobre consumo dietario se caracterizó por el alto grado de precisión logrado en la determinación de los tamaños de porción, a través de modelos de alimentos abstractos, validados en las poblaciones estudiadas y ligados a la tabla de composición de alimentos con que se codificó el consumo dietario $(28,29)$. Los detalles del diseño de la muestra y su selección ya fueron publicados (30). Los 822 sujetos dieron origen a $1616 \mathrm{R} 24 \mathrm{H}$.

Fuentes de información. Dos cuestionarios fueron aplicados a todos los integrantes del hogar; un formato para recoger información sociodemográfica y biológica del individuo y dos R24H (31). Los R24H fueron aplicados a todos los integrantes del hogar de manera repetida con intervalo mínimo de una semana para evitar respuestas con base en la memoria $(7,8)$. La inseguridad alimentaria del hogar donde habita el sujeto se estableció con la escala ELCSA aplicada al jefe del hogar $(31,32)$. El nivel socioeconómico de los sujetos se estableció a través de tres variables proximales; el sistema de identificación y clasificación de potenciales beneficiarios para programas sociales (SISBEN) (33), el tipo de afiliación a la seguridad social y el nivel de inseguridad alimentaria del hogar. La ingesta usual de energía fue estimada para cada sujeto con base en los métodos propuesto por la universidad de lowa (34) y utilizando PC-Side (35).

Análisis estadístico. Para corregir la asimetría de las variables nutricionales $(\mathrm{Vn})$, primero se estimó la densidad de cada una de ellas por 1000 kilocalorías y luego fue transformada a $(\mathrm{Vn})^{0,5}$. La descripción del consumo de energía por las características biológicas y sociodemográficas de los sujetos se realizó con base en la ingesta usual a través del promedio y su error estándar. Las diferencias ajustadas del consumo de energía y sus respectivos intervalos de confianza del 95\% (IC 95\%) se calcularon con base en modelos de regresión lineal con el consumo de kilocalorías usuales como la variable dependiente y el sexo, la edad (continua), el IMC, la escolaridad, el nivel de actividad física, la seguridad social, el nivel de SISBEN, consumir alimentos fuera de la casa, el área (urbano/rural), y el nivel de inseguridad/seguridad alimentaria en el hogar, como explicatorias. Para las ciudades estudiadas el modelo no incluyó el área geográfica por su colinealidad. La estimación de los coeficientes de variación "intra" (CVw) y "entre" (CVb) para cada variable nutricional, se realizó con base en análisis de varianza (ANOVA), considerando el día de la ingesta como una medida repetida. La estimación de la varianza "intra" $\left(S^{2} w\right)$ y "entre" ( $\left.\mathrm{S}^{2} \mathrm{~b}\right)$, se realizó con base en ANOVA. El cálculo de los coeficientes de variación y las varianzas $S^{2} w$ y $S^{2}$ b se realizó con el fin de establecer la viabilidad al estudiar las variables nutricionales en relaciones dieta-eventos de salud y enfermedad, también para estimar tamaños muestrales y el número mínimo de días en que un sujeto debe registrar su dieta, para aproximarse con diferentes grados de error, al verdadero valor medio de la ingesta de nutrientes específicos. El cálculo del número de repeticiones por sujeto o número de registros por día, necesarios para estimar el verdadero valor medio de la ingesta de un nutriente, con diferentes grados de error fue calculado como (7, 8, 36);

$$
\mathrm{n}=[(\mathrm{Z} \infty * \mathrm{CVw}) / \mathrm{Do}]^{2} \text {, donde }
$$

$\mathrm{n}=$ el número de días necesarios por persona;

$Z \infty=$ la desviación normal, para estar dentro de un rango de confiabilidad alrededor del verdadero promedio, dado un grado de error (Do);

$C V w=$ el coeficiente de variación intra-persona, expresado en porcentaje y

Do = el limite específico de error (como un porcentaje alrededor de la verdadera ingesta, en el tiempo).

El análisis estadístico se realizó con el software Stata/SE versión 13,1 (Stata Corporation, College Station, TX) (37). El estudio fue aprobado por el Comité de Etica en Salud de la Universidad Industrial de Santander en Bucaramanga, Colombia.

\section{Resultados.}

Un total de 822 sujetos entre 18 y 59 años participaron, 473 fueron mujeres (56\%). Doscientos noventa y seis sujetos residían en Bogotá (36\%), 182 en Bucaramanga y su Area Metropolitana (22\%), 168 en Tenjo (20\%) y 176 en Sibaté (22\%). Los sujetos entre 18 y 29 años aportaron su información dietaria en 528 R24H, los sujetos entre 30 y 59 años en 1088 R24H. Todos los sujetos contestaron dos R24H, excepto 23 en los cuales ni la edad media o el sexo fueron diferentes al contrastarla con los que contestaron, $p=0,588$ y $p=0,463$ respectivamente. El tiempo medio entre las aplicaciones de los $\mathrm{R} 24 \mathrm{H}$ fue 16 días (IC 95\%: 14 a 17). La edad media de los sujetos estudiados fue 36,8 años (IC 95\%: 36,0 a 37,6), sin diferencia por sexo, $\mathrm{p}=0,918$. El $47,7 \%$ de los sujetos presentaba exceso de peso, $61,3 \%$ de las mujeres y $38,7 \%$ de los hombres, $p=0,007$. Por cada cinco años de envejecimiento, el consumo de kilocalorías se reduce, en promedio -27 (IC 95\%: -44 a -11). Las variables asociadas el consumo de energía fueron la edad agrupada, $\mathrm{p}<0,0001$, el tipo de afiliación a la seguridad social, $p=0,023$, el nivel de seguridad alimentaria del hogar, $\mathrm{p}=<0,0001$, y el área geográfica, $p=0,003$. En la zona rural es evidente la desventaja socioeconómica, dado el nivel de seguridad alimentaria y de SISBEN, $p<0,0001$ para ambas. Otras características sociodemográficas y en relación con el consumo de energía se presentan en la tabla 1.

La asimetría positiva de las variables nutricionales $(\mathrm{Vn})$, fue corregida en buena medida al transformarlas. De los 27 nutrientes estudiados en la zona rural el CVw estuvo entre $0 \%$ y $100 \%$ en 19 , entre $101 \%$ y $200 \%$ en 3 nutrientes (potasio, magnesio y cobre) y por encima de $201 \%$ en 5 nutrientes (vitamina A, acido pantoténico, vitamina B6, ácido fólico, vitamina B12). En la zona urbana el $\mathrm{CVw}$ estuvo entre 0 y 
TABLA 1

Ingesta usual/día de calorías en adultos colombianos (18 a 59 años) de acuerdo con condiciones sociodemográficas.



a Los totales pueden ser $<822$ por valores perdidos.

b Test de tendencia lineal para los predictores ordinales. Para el sexo, la actividad física, el nivel de SISBEN, la seguridad social, recibir apoyo alimentario, consumir alimentos fuera de la casa, el área geográfica y la ciudad estudiada, P es con base en análisis de varianza (ANOVA).

c Las diferencias fueron ajustadas en un modelo de regresión lineal, con el consumo de kilocalorías usuales como la variable dependiente y el sexo, la edad (continua), el IMC, la escolaridad, el nivel de actividad física, la seguridad social, el nivel de SISBEN, consumir alimentos fuera de la casa, el área (urbano/rural), y el nivel de inseguridad/seguridad alimentaria en el hogar, como explicatorias. Para las ciudades estudiadas el modelo no incluyo el área geográfica por su colinealidad.

d Test ajustado para tendencia lineal o ANOVA para los predictores ordinales o categóricos.

e Con base en los valores del índice de masa corporal (IMC); kg/mt2.

f Con base en el sistema de identificación y clasificación de potenciales beneficiarios para programas sociales (SISBEN) (33).

g Con base en una versión modificada de la ELCSA (Sin los ítems 9 y 13) $(31,32)$. 
$100 \%$ en 18 nutrientes, entre $101 \%$ y $200 \%$ en 8 (sodio, potasio, magnesio, cobre, ácido pantoténico, vitamina B6, ácido fólico, vitamina B12) y por encima de $201 \%$ en un nutriente (vitamina A). Tanto para la zona urbana y rural el menor CVw fue para los carbohidratos. El CVb fue tanto para la zona rural como la urbana mayor en todos los nutrientes. Siete nutrientes en la zona rural alcanzaron CVb entre 0\% y 100\% (grasa polinsaturada, colesterol, sodio, potasio, zinc, tiamina, riboflavina), y seis por encima de $200 \%$ (magnesio, vitamina A, ácido pantoténico, vitamina B6, ácido fólico y vitamina B12). En la zona urbana seis nutrientes entre $100 \%$ y $200 \%$ (sodio, potasio, zinc, tiamina, riboflavina, niacina), seis nutrientes por encima de $201 \%$ (magnesio, cobre, vitamina A, ácido pantoténico, vitamina B6, ácido fólico, vitamina B12). Tanto para la zona urbana y rural el mayor CVb fue para la Vitamina B12. Los detalles pueden observarse en la tabla 2.

La razón entre varianzas $\left(\mathrm{S}^{2} \mathrm{w} / \mathrm{S}^{2} \mathrm{~b}\right)$ fue consistentemente mayor en la zona rural, excepto para la energía y el sodio. El calcio en la zona rural y el cobre en la urbana presentaron la menor razón $\mathrm{S}^{2} \mathrm{w} / \mathrm{S}^{2} \mathrm{~b}, 0,36$ y 0,12 respectivamente. La vitamina
A en la zona rural y el sodio en la urbana, la mayor razón, 0,98 y 0,91 respectivamente (tabla 3 ).

La tabla 4 presenta las fuentes de variación y su aporte relativo en nueve nutrientes específicos. En los grupos de edad entre 18-29 años y 30-59, el nivel socioeconómico y el área geográfica son los principales determinantes de la variabilidad. Sin embargo, para cada nutriente hay particularidades, por ejemplo, en el consumo de energía el sexo, es la principal fuente de variación o en grupo de 18-29 años la escolaridad es una fuente de variación importante para el colesterol, mientras que para el hierro o la vitamina A en el mismo grupo de edad el aporte de la escolaridad a su variabilidad es despreciable.

El número de días necesario para estudiar con niveles aceptables de error el consumo dietario es mayor para la vitamina A y el colesterol en relación con los otros estudiados, hasta el punto de hacer poco viable el estudio de estos nutrientes en el establecimiento de relaciones dieta-eventos de salud y enfermedad a través del recordatorio de 24 horas. Para los macronutrientes son necesarios hasta cinco mediciones repetidas para obtener el verdadero promedio de la ingesta de éstos con

\section{TABLA 2}

Ingesta promedio diaria de nutrientes en adultos colombianos entre 18 y 59 años en áreas con diferente grado de urbanismo y coeficientes (\%) de variación intra-persona (CVw) y entre-personas (CVb).

\begin{tabular}{|c|c|c|c|c|c|c|c|}
\hline & \multirow{2}{*}{$\begin{array}{l}\text { Número de días } \\
\text { Nutriente }^{\mathrm{b}}\end{array}$} & \multicolumn{3}{|c|}{$\begin{array}{c}\text { Rural } \\
677\end{array}$} & \multicolumn{3}{|c|}{$\begin{array}{c}\text { Urbano } \\
939\end{array}$} \\
\hline & & $\mathrm{X} \pm \mathrm{DS}$ & $C V w$ & $\mathrm{CVb}$ & $\mathrm{X} \pm \mathrm{DS}$ & $C V w$ & $\mathrm{CVb}$ \\
\hline & Kilocalorías ${ }^{*}$ & $2121 \pm 101$ & 16,1 & 26,3 & $1901 \pm 88$ & 17,2 & 25,0 \\
\hline & Proteínas $(\mathrm{g})$ * & $29,3 \pm 0,7$ & 14,1 & 17,2 & $34,8 \pm 0,8$ & 13,1 & 16,7 \\
\hline & Grasa total $(g) *$ & $24,9 \pm 1,2$ & 18,1 & 25,5 & $31,1 \pm 1,0$ & 14,5 & 21,1 \\
\hline & Grasa saturada $(g) *$ & $2,0 \pm 1,2$ & 69,6 & 88,1 & $4,6 \pm 1,1$ & 36,9 & 60,7 \\
\hline & Grasa monoinsaturada $(\mathrm{g})$ * & $1,9 \pm 1,2$ & 71,7 & 90,1 & $4,8 \pm 1,1$ & 35,6 & 57,0 \\
\hline & Grasa poliinsaturada $(\mathrm{g}) *$ & $1,0 \pm 0,8$ & 82,1 & 100,5 & $3,5 \pm 1,3$ & 43,3 & 72,7 \\
\hline & Colesterol $(\mathrm{mg}) *$ & $28,2 \pm 30,9$ & 91,1 & 116,9 & $46,9 \pm 25,1$ & 60,9 & 84,2 \\
\hline & Carbohidratos $(\mathrm{g}) *$ & $162,5 \pm 1,9$ & 8,7 & 12,5 & $141,8 \pm 1,7$ & 8,7 & 12,7 \\
\hline & Fibra cruda $(g) *$ & $2,0 \pm 0,3$ & 34,0 & 46,0 & $1,5 \pm 0,4$ & 46,3 & 59,2 \\
\hline & Cenizas $(g)+$ & $5,5 \pm 1,4$ & 48,5 & 52,3 & $6,0 \pm 1,1$ & 37,4 & 46,7 \\
\hline & Calcio $(\mathrm{mg}) *$ & $230,5 \pm 16,2$ & 19,2 & 32,1 & $301,7 \pm 30,9$ & 22,2 & 39,1 \\
\hline & Fosforo $(\mathrm{mg}) *$ & $105,9 \pm 56,3$ & 63,0 & 81,2 & $244,9 \pm 57,2$ & 30,7 & 60,7 \\
\hline & Hierro (mg) & $7,8 \pm 0,6$ & 24,6 & 30,9 & $8,4 \pm 1,8$ & 22,8 & 60,7 \\
\hline & Sodio $(\mathrm{mg})^{+}$ & $619,9 \pm 589,8$ & 90,8 & 103,6 & $448,1 \pm 514,6$ & 104,7 & 109,3 \\
\hline & Potasio $(\mathrm{mg}) *$ & $10,0 \pm 24,7$ & 140,5 & 171,9 & $19,1 \pm 42,4$ & 114,0 & 176,4 \\
\hline & Magnesio (mg) * & $1,2 \pm 5,1$ & 165,8 & 235,6 & $4,0 \pm 17,1$ & 108,5 & 271,1 \\
\hline & Zinc $(m g) *$ & $1,0 \pm 0,8$ & 72,0 & 108,2 & $2,3 \pm 2,2$ & 48,4 & 126,1 \\
\hline & Cobre $(\mathrm{mg}) *$ & $0,0 \pm 0,1$ & 190,9 & 100,0 & $0,04 \pm 0,3$ & 123,1 & 350,9 \\
\hline & Vitamina A (Retinol) * & $0,8 \pm 6,1$ & 271,1 & 276,7 & $2,5 \pm 18,2$ & 245,0 & 292,2 \\
\hline & Tiamina $(m g) *$ & $0,1 \pm 0,1$ & 67,6 & 106,0 & $0,3 \pm 0,2$ & 44,6 & 108,6 \\
\hline & Ribloflavina (mg)* & $0,2 \pm 0,1$ & 64,1 & 102,6 & $0,4 \pm 0,3$ & 40,6 & 102,5 \\
\hline & Niacina $(\mathrm{mg}) *$ & $2,2 \pm 1,5$ & 68,7 & 95,5 & $5,0 \pm 3,1$ & 44,6 & 101,4 \\
\hline & Ácido pantoténico $(\mathrm{mg})$ * & $0,0 \pm 0,4$ & 257,9 & 390,3 & $0,2 \pm 1,5$ & 141,5 & 396,3 \\
\hline & Vitamina B6 $(\mathrm{mg}) *$ & $0,0 \pm 0,1$ & 240,0 & 346,4 & $0,0 \pm 0,3$ & 133,3 & 349,9 \\
\hline & Ácido fólico (mcg) * & $1,3 \pm 15,5$ & 255,7 & 409,2 & $7,1 \pm 61,9$ & 142,9 & 390,7 \\
\hline & Vitamina B12 $(\mathrm{mcg}) *$ & $0,0 \pm 0,2$ & 271,4 & 416,5 & $0,1 \pm 1,0$ & 153,1 & 411,0 \\
\hline & Ácido ascórbico (mg) & $29,9 \pm 11,1$ & 54,5 & 66,9 & $32,8 \pm 18,1$ & 56,4 & 88,1 \\
\hline & \multicolumn{7}{|c|}{$\begin{array}{l}\text { Número de días en los que se basó el cálculo; el método de recolección de la ingesta dietaria fue recordatorio del consumo de las últimas } 24 \\
\text { horas. }\end{array}$} \\
\hline & \multicolumn{7}{|c|}{ Con base en valores transformados; (Densidad de nutrientes por 1000 kilocalorías) 0,5 } \\
\hline & \multicolumn{7}{|c|}{$\begin{array}{l}\text { Con base en valores transformados; (Densidad de nutrientes por } 1000 \text { kilocalorías) 0,5 } \\
\text { Test para la diferencia de medias entre áreas geográficas; }{ }^{*} p<0,0001 .+p<0,05 .+p<0,001\end{array}$} \\
\hline
\end{tabular}


niveles de error entre $15 \%$ y $40 \%$. Como es predecible a menor $\mathrm{CVw}$, es más viable el estudio del nutriente (tabla 5).

\section{Discusión}

El primer aspecto a discutir, es que todos los CVw son en términos absolutos menores que los $\mathrm{CVb}$ tanto en el área rural como la urbana y que, independientemente del área geográfica los CV son específicos, cada Vn se comporta de manera particular. Razones $\mathrm{Sw}^{2} / \mathrm{Sb}^{2}$ cercanas a 0 permiten con mayor probabilidad encontrar de existir, asociaciones del consumo dietario con eventos de salud enfermedad, por el contrario razones cercanas a 1 o superiores, lo dificultan, fenómeno conocido como atenuación o dilución de la relación (7). En niños, donde la exploración y consumo de alimentos aumenta conforme participan de la alimentación familiar y luego de la escolar y social, es común encontrar razones $\mathrm{Sw}^{2} / \mathrm{Sb}^{2}$ mayores de 1, lo que dificultad encontrar asociaciones epidemiológicas (7, 8, 21-27, 36). Nosotros estudiamos sujetos adultos y en el anterior sentido con excepción de la vitamina $\mathrm{A}$, todas las razones $\mathrm{Sw}^{2} / \mathrm{Sb}^{2}$ se encuentran dentro de valores $<1$, lo que permitiría aún el desarrollo de la epidemiología nutricional en Colombia.
En contra de lo esperado, tanto en la zona rural como la urbana, la razón $\mathrm{Sw}^{2} / \mathrm{Sb}^{2}$ en el grupo de 18-29 años fue con algunas excepciones, menor que en el grupo de 30-59 años, lo anterior es debido a que en el grupo de edad de 18-29 años la $\mathrm{Sb}^{2}$ es mayor en términos relativos frente a los sujetos entre 30-59 años, mientras la $\mathrm{Sw}^{2}$ es menor, en la medida que en los más jóvenes aún no terminan la etapa de construcción y consolidación de hábitos alimentarios. En otras palabras, lo hábitos alimentarios de sujetos entre 30-59 años se traducen en mayor $\mathrm{Sw}^{2}$ relativa con los más jóvenes y mejor discriminación $\mathrm{Sb}^{2}$ entre ellos, aunque menor en términos relativos con los de 18-29 años. Como ya se había discutido la variabilidad "intra" fue consistentemente mayor en la zona rural, y ante mejor discriminación entre sujetos por dietas más diversas en alimentos en la zona urbana, las razones $\mathrm{Sw}^{2} / \mathrm{Sb}^{2}$ con excepción de la energía y el sodio, fueron consistentemente mayores en la zona rural (tabla 3).

Dado que la variabilidad de la dieta está íntimamente ligada al nivel de desarrollo de las sociedades, y al interior de Colombia se evidencian altos niveles de desigualdad, inequidad y desarrollo diferencial entre áreas geográficas $(1,2)$, conjuntamente con cambios en los patrones alimentarios $(19,38)$, el

TABLA 3

Razón de los componentes de la varianza "intra-persona" y "entre-personas" ( $\left.\mathrm{S}^{2} \mathrm{w} / \mathrm{S}^{2} \mathrm{~b}\right)$, para la ingesta de nutrientes observada en adultos colombianos entre 18 y 59 años en áreas con diferente grado de urbanismo

\begin{tabular}{|c|c|c|c|c|c|c|c|}
\hline \multirow[b]{2}{*}{ Nutrientes ${ }^{b}$} & \multicolumn{3}{|c|}{ Rural } & \multicolumn{3}{|c|}{ Urbano } & \multirow[b]{2}{*}{$U-R^{a}$} \\
\hline & $\begin{array}{c}18-29 \\
{[231] \mathrm{c}}\end{array}$ & $\begin{array}{l}30-59 \\
{[446]}\end{array}$ & $\begin{array}{l}\text { Total } \\
{[677]}\end{array}$ & $\begin{array}{l}18-29 \\
{[297]}\end{array}$ & $\begin{array}{c}30-59 \\
{[642]}\end{array}$ & $\begin{array}{l}\text { Total } \\
\text { [939] }\end{array}$ & \\
\hline Kilocalorías & 0,37 & 0,38 & 0,37 & 0,40 & 0,50 & 0,47 & $+0,10$ \\
\hline Proteínas (g) & 0,69 & 0,72 & 0,71 & 0,60 & 0,64 & 0,62 & $-0,09$ \\
\hline Grasa total (g) & 0,37 & 0,59 & 0,52 & 0,49 & 0,49 & 0,48 & $-0,04$ \\
\hline Grasa saturada (g) & 0,41 & 0,85 & 0,66 & 0,53 & 0,33 & 0,39 & $-0,27$ \\
\hline Grasa monoinsaturada (g) & 0,39 & 0,89 & 0,67 & 0,50 & 0,35 & 0,39 & $-0,28$ \\
\hline Grasa poliinsaturada (g) & 0,48 & 0,80 & 0,68 & 0,41 & 0,35 & 0,36 & $-0,32$ \\
\hline Colesterol (mg) & 0,46 & 0,75 & 0,63 & 0,63 & 0,49 & 0,53 & $-0,10$ \\
\hline Carbohidratos (g) & 0,31 & 0,67 & 0,52 & 0,46 & 0,49 & 0,48 & $-0,04$ \\
\hline Fibra cruda (g) & 0,75 & 0,51 & 0,55 & 0,55 & 0,70 & 0,62 & $+0,07$ \\
\hline Cenizas (g) & 0,74 & 0,91 & 0,85 & 0,73 & 0,60 & 0,65 & $-0,20$ \\
\hline Calcio (mg) & 0,47 & 0,30 & 0,36 & 0,23 & 0,44 & 0,33 & $-0,03$ \\
\hline Fosforo (mg) & 0,44 & 0,74 & 0,62 & 0,29 & 0,25 & 0,26 & $-0,36$ \\
\hline Hierro (mg) & 0,43 & 0,74 & 0,61 & 0,08 & 0,28 & 0,14 & $-0,47$ \\
\hline Sodio (mg) & 0,64 & 0,97 & 0,80 & 0,89 & 0,90 & 0,91 & $+0,11$ \\
\hline Potasio (mg) & 0,91 & 0,57 & 0,67 & 0,32 & 0,50 & 0,42 & $-0,25$ \\
\hline Magnesio (mg) & 0,37 & 0,64 & 0,50 & 0,11 & 0,24 & 0,16 & $-0,34$ \\
\hline Zinc (mg) & 0,29 & 0,62 & 0,45 & 0,11 & 0,21 & 0,15 & $-0,30$ \\
\hline Cobre(mg) & 0,24 & 0,66 & 0,41 & 0,07 & 0,18 & 0,12 & $-0,29$ \\
\hline Vitamina A (Retinol) & 1,17 & 0,90 & 0,98 & 0,63 & 0,73 & 0,70 & $-0,28$ \\
\hline Tiamina (mg) & 0,29 & 0,50 & 0,38 & 0,11 & 0,23 & 0,16 & $-0,22$ \\
\hline Ribloflavina (mg) & 0,29 & 0,54 & 0,43 & 0,12 & 0,22 & 0,16 & $-0,27$ \\
\hline Niacina (mg) & 0,40 & 0,68 & 0,55 & 0,13 & 0,29 & 0,19 & $-0,36$ \\
\hline Ácido pantoténico (mg) & 0,26 & 0,67 & 0,44 & 0,08 & 0,20 & 0,13 & $-0,31$ \\
\hline Vitamina B6 (mg) & 0,33 & 0,66 & 0,50 & 0,09 & 0,22 & 0,15 & $-0,35$ \\
\hline Ácido fólico (mcg) & 0,25 & 0,59 & 0,40 & 0,09 & 0,20 & 0,13 & $-0,27$ \\
\hline Vitamina B12 (mcg) & 0,29 & 0,68 & 0,45 & 0,09 & 0,21 & 0,14 & $-0,31$ \\
\hline Ácido ascórbico (mg) & 0,62 & 0,64 & 0,65 & 0,26 & 0,56 & 0,40 & $-0,25$ \\
\hline
\end{tabular}

a Diferencia; [Total Urbano (U)-Total Rural (R)]

b Con base en valores transformados; (Densidad de nutrientes por 1000 kilocalorías) 0,5

c [n]; Número de días en los que se basó el cálculo; el método de recolección de la ingesta dietaria fue recordatorio del consumo de las últimas 24 horas. 
análisis fue enfocado para diferenciar la variabilidad de la dieta entre diferentes niveles de desarrollo estructural y socioeconómico, el rural y el urbano (39).

La apertura de mercados se ha traducido en mayor disponibilidad y variabilidad en la oferta de alimentos que en teoría se traduce en dietas más heterogéneas. Estos cambios son evidentes en las zonas urbanas, y en muchas zonas rurales. Sin embargo, la incorporación de dietas variadas en reemplazo de dietas monótonas o con poca variabilidad de alimentos, la denominada tradicional, no sólo depende del mercado, en estas decisiones complejas de elección alimentaria, intervienen entre otros factores la cultura, la ecología, el nivel de escolaridad y la capacidad adquisitiva $(15,16)$. El aspecto económico es un determinante de la variabilidad de la dieta, se ha demostrado, que en grupos sociales pobres y sociedades no industrializadas como muchas de las rurales en Colombia, donde el consumo está íntimamente ligado al ingreso, la $\mathrm{Sw}^{2}$ es mayor en términos relativos que en sociedades con mayor riqueza y desarrollo ( 7 , 27). En Vn cuyo consumo dietario está altamente ligado al ingreso como el colesterol, la grasa poliinsaturada, las proteínas, el ácido fólico y la vitamina B12, el CVw fue mayor en la zona rural, lo que corrobora que en condiciones de pobreza comparadas contra otras menos pobres, en las dietas monótonas o con poca diversidad de alimentos, las variaciones en el consumo se expresan como mayor variabilidad "intra" sujeto $(7,27)$. Corroborando lo anterior, dado que el consumo de carbohidratos no es dependiente del ingreso, el CVw para el consumo de carbohidratos no es diferencial en las áreas geográficas. Complementariamente, con pocas excepciones el consumo medio de nutrientes es menor en la zona rural a pesar de consumirse más energía en términos absolutos que en la zona urbana, otra expresión de la baja calidad de la dieta de sujetos con menos desarrollo estructural y mayor pobreza. Nuestros hallazgos confirman lo anterior, pues con excepción de dos variables nutricionales, energía y sodio, todas las razones $\mathrm{Sw}^{2} / \mathrm{Sb}^{2}$ son mayores en la zona rural. En áfrica un estudio llevado a cabo en mujeres de zonas rurales marginadas con dietas muy limitadas, se encontró el mismo efecto reportado aquí, mayor variabilidad $\mathrm{Sw}^{2}$ en el área rural que en la urbana (27). Sin embargo, otro estudio realizado en adultos canadienses, mostró el efecto del otro extremo del desarrollo estructural y económico sobre la variabilidad intra $(\mathrm{CVw})$, en él éste es consistente mayor que el $\mathrm{CVb}$ (26), lo aquí reportado muestra como el CVw es consistentemente menor que el $\mathrm{CVb}$ tanto en la zona rural como urbana.

En niños se ha reportado que a medida que aumenta la edad aumenta la variabilidad que ésta aporta al consumo ( 7 , 40), sin embargo, la edad aunque aporta a la variabilidad no es la principal fuente de la misma. Las principales fuentes de variabilidad fueron el sexo, y todas las proximales del nivel socioeconómico incluído el área geográfica. Ni la actividad física, la edad o el día de la semana son fuentes importantes de variabilidad. En las discusiones teóricas, el día de la semana en que se hace un $\mathrm{R} 24 \mathrm{H}$ o si se realiza en días consecutivos, son variables que han sido consideradas como relevantes por su efecto sobre la variabilidad en el consumo. Se argumenta a favor y en contra de la cultura alimentaria en los fines de semana y sobre la correlación que existe entre días consecutivos del consumo, en otros términos, sobre el nivel de independencia entre el consumo dietario realizado en días consecutivos $(7,22)$, transcurrieron en promedio 16 días entre una y otra aplicación de los R24H. En Colombia existe evidencia de que el día de la semana en que se mide la ingesta se comporta como una medición independiente, incluso en días consecutivos y fines de semana (22). En Colombia algo que puede ser considerado a favor de la viabilidad del estudio de las relaciones dieta-eventos de salud enfermedad, es que para todos los macro y micro nutrientes estudiados, la variación residual o "intra" estuvo entre $0,7 \%$ y $9,6 \%$ (tabla 4 ).

La viabilidad del estudio de las relaciones dieta-eventos de salud enfermedad está en concordancia con el número de días que son necesarios para medir una exposición dietaria con precisión, para casi todos los nutrientes estudiados esto es posible como ha sido reportado por otros autores para el colesterol y la vitamina A, es cada vez más inviable (23-26). El hierro presentó un comportamiento contrario al esperado, pues es un nutriente del que también se reporta la dificultad de su medición por su alta variabilidad lo que obliga a muchas repeticiones, nuestro resultado puede deberse a que la medición de sólo dos días puede homogenizar artificialmente el consumo o la falta del mismo, dados los altos niveles de anemia ferropénica reportados en Colombia (1) ésta sería la explicación más plausible. El no contar con suficientes biomarcadores del consumo dietario es un reto aún vigente.

Para los macronutrientes, energía, proteínas, grasa total, y carbohidratos, pocas repeticiones de la medición dietaria, permiten con un mínimo de error aproximarse al verdadero promedio. Para las vitaminas y micronutirentes dada su variabilidad intra sujeto son necesarias más repeticiones o en su reemplazo, estudiar los metabolitos circulantes en sangre, lo que exige un balance entre recursos financieros, aspectos logísticos y capacidad tecnológica, no siempre a favor de los metabolitos dadas las limitadas condiciones socioeconómicas de los financiadores y a veces tecnológicas en los laboratorios regionales. Consistentemente con los hallazgos reportados y hasta ahora discutidos, en la zona rural para los nutrientes socioeconómicamente más dependientes como las proteínas, la grasa, el colesterol, la niacina y la vitamina A, es más complejo su estudio por requerirse de mayor número de repeticiones para acercarse a su verdadero promedio con un nivel de error tolerable.

Los estudios sobre la variabilidad de la dieta en América Latina son escasos, de los dos identificados en Colombia, sólo uno -reportado en 2006- presenta valores similares en las $\mathrm{Sw}^{2} /$ $\mathrm{Sb}^{2}$ y en las fuentes de variabilidad, a los aquí reportados (22, 24). El otro realizado en mujeres universitarias jóvenes, reporta CV extremadamente altos y no es posible la comparación sobre las fuentes de variabilidad ya que no se estimaron. En él se concluye que "....El principal hallazgo fue la presencia de heterocedasticidad entre las varianzas de las ingestas de energía y los diferentes nutrientes analizados: siendo incorrectos, para este grupo poblacional, los esquemas de recolección de información del consumo aplicados usualmente en la práctica clínica y la investigación epidemiológica" (24). La presencia de heterocedasticidad de las varianzas, presupone que son incorrectas las prácticas clínicas y epidemiológicas actuales cuando se trata de medir la dieta y su relación con eventos de salud enfermedad (24, 41).

Alcances y limitaciones. Como fortalezas del estudio pueden mencionarse que; a) se basó en mediciones del consumo dietario realizadas con $\mathrm{R} 24 \mathrm{H}$ con intervalo medio entre ellos de 16 días. Está reportado que la medición en días consecutivos podría llevar a subestimación de la $\mathrm{Sw}^{2}(7)$, b) que la estimación de los tamaños de porción se realizó con modelos abstractos previamente validados lográndose mayor precisión en la estimación del consumo, c) se basa en un tamaño muestral grande y más de 1600 R24H con alta calidad, d) se realizó en áreas con diferentes niveles de desarrollo económico y estructural y e) el diseño muestral y de selección de sujetos en buena medida representa otras áreas y sujetos del país. Como limitaciones 
podemos mencionar las propias de las tablas de composición de alimentos, aspecto aún no superado del todo en la epidemiología nutricional $(7,8)$, que podría llevar a subestimación y sobreestimación de las cantidades consumidas y por supuesto a modificación de la variabilidad, en particular para algunos micronutrientes y las propias del método R24H que aun cuando no es un estándar de oro, si es el más utilizado en la estimación del consumo en la investigación epidemiológica $(7,8,36)$.

TABLA 4

Fuentes relativas de variación (\%) para ingesta diaria de nutrientes. (Sujetos, variación "entre-sujetos" (S²b); residual, variación "intra-persona" $\left(\mathrm{S}^{2} \mathrm{~W}\right)$.

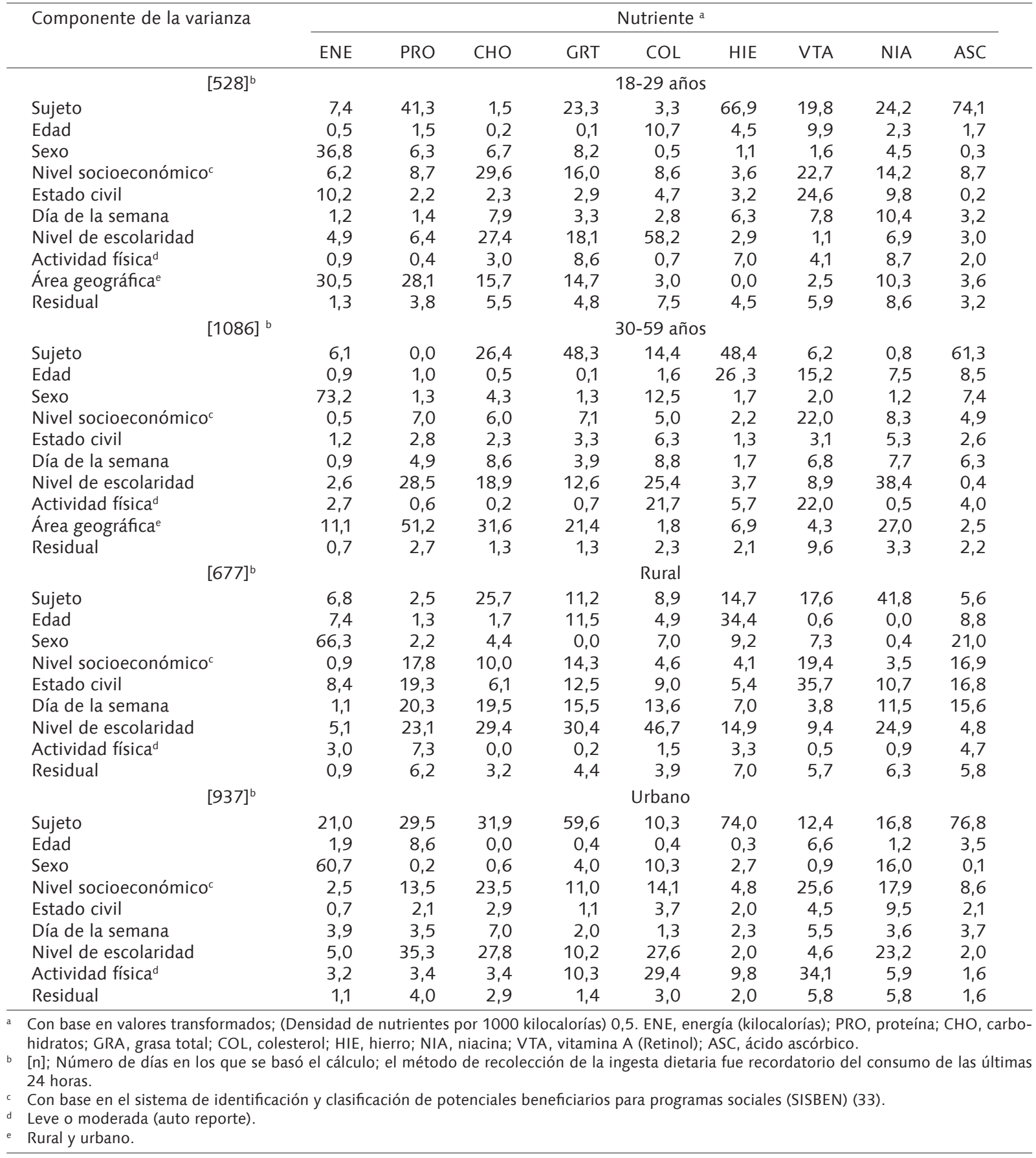


En conclusión se pudo establecer que el mayor consumo de energía en la zona rural es una expresión más de la baja calidad de la dieta en relación con la densidad de nutrientes que se observa en la dieta urbana, que los cambios en la alimentación que experimentan sujetos colombianos en presencia de doble carga nutricional $(42,43)$ y del actual contexto y características de desarrollo económico y estructural pueden considerarse como de transición alimentaria (19) y se traducen en términos de variabilidad de la dieta en mayor $\mathrm{Sw}^{2} / \mathrm{Sw}^{2}$ en las zonas rurales por vía de la pobreza y en términos más generales, en mayor variabilidad entre sujetos $\left(\mathrm{Sb}^{2}\right)$ que Ileva a $\mathrm{Sw}^{2} /$ $\mathrm{Sb}^{2}$ menores de 1, lo que permite aún, de manera viable y con pocas excepciones (vitamina A y colesterol), el desarrollo de la epidemiología nutricional y el estudio de las relaciones entre dieta-eventos de salud enfermedad en Colombia.
Declaración de conflicto de interés.

Los autores declaran que no tienen conflicto de interés de ningún tipo, ni real o potencial sobre los resultados presentados.

\section{Financiación}

Este estudio fue posible por la cofinanciación del Departamento Administrativo de Ciencia, Tecnología e Innovación de Colombia (COLCIENCIAS), código; 110245921548 y de la Secretaria Distrital de Salud de Bogotá, la Gobernación de Cundinamarca, y las Universidades Nacional de Colombia e Industrial de Santander. Código interno UIS; 8677.

\section{RESUMEN}

Durante 2009-2011, se recolectó información sobre el consumo dietario de 822 sujetos entre 18-59 años de edad.

TABLA 5

Número de días necesarios por sujeto, para obtener un valor promedio de la ingesta de algunos nutrientes dentro del verdadero promedio, con un $95 \%$ de confianza; $(Z=1,96)$.

\begin{tabular}{|c|c|c|c|c|c|c|c|}
\hline \multirow[b]{2}{*}{ Nutriente a } & \multirow[b]{2}{*}{$C V w b$} & \multicolumn{6}{|c|}{ Error alrededor del verdadero promedio } \\
\hline & & $5 \%$ & $10 \%$ & $15 \%$ & $20 \%$ & $30 \%$ & $40 \%$ \\
\hline \multicolumn{8}{|l|}{ Kilocalorías } \\
\hline Rural & 16,1 & 40 & 10 & 4 & 2 & 1 & 1 \\
\hline Urbano & 17,2 & 45 & 11 & 5 & 3 & 1 & 1 \\
\hline Total & 16,8 & 43 & 11 & 5 & 3 & 1 & 1 \\
\hline \multicolumn{8}{|l|}{ Proteína (g) } \\
\hline Rural & 14,1 & 31 & 36 & 22 & 5 & 0 & 0 \\
\hline Urbano & 13,1 & 26 & 27 & 12 & 1 & 0 & 0 \\
\hline Total & 13,5 & 28 & 30 & 15 & 2 & 0 & 0 \\
\hline \multicolumn{8}{|l|}{ Carbohidratos (g) } \\
\hline Rural & 8,7 & 12 & 3 & 1 & 1 & 0 & 0 \\
\hline Urbano & 8,7 & 12 & 3 & 1 & 1 & 0 & 0 \\
\hline Total & 8,7 & 12 & 3 & 1 & 1 & 0 & 0 \\
\hline \multicolumn{8}{|l|}{ Grasa total (g) } \\
\hline Rural & 18,1 & 50 & 13 & 6 & 3 & 1 & 1 \\
\hline Urbano & 14,5 & 32 & 8 & 4 & 2 & 1 & 1 \\
\hline Total & 16,0 & 39 & 10 & 4 & 2 & 1 & 1 \\
\hline \multicolumn{8}{|l|}{ Colesterol (mg) } \\
\hline Rural & 91,1 & 1275 & 319 & 142 & 80 & 35 & 20 \\
\hline Urbano & 60,9 & 570 & 142 & 63 & 36 & 16 & 9 \\
\hline Total & 71,6 & 788 & 197 & 88 & 49 & 22 & 12 \\
\hline \multicolumn{8}{|l|}{ Hierro (mg) } \\
\hline Rural & 24,6 & 80 & 20 & 9 & 5 & 2 & 1 \\
\hline Urbano & 22,8 & 93 & 23 & 10 & 6 & 3 & 1 \\
\hline Total & 23,5 & 85 & 21 & 9 & 5 & 2 & 1 \\
\hline \multicolumn{8}{|l|}{ Niacina (mg) } \\
\hline Rural & 68,7 & 725 & 181 & 81 & 45 & 20 & 11 \\
\hline Urbano & 46,6 & 334 & 83 & 37 & 21 & 9 & 5 \\
\hline Total & 52,9 & 430 & 108 & 48 & 27 & 12 & 7 \\
\hline \multicolumn{8}{|c|}{ Vitamina A (Retinol) } \\
\hline Rural & 271,1 & 11294 & 2823 & 1255 & 706 & 314 & 176 \\
\hline Urbano & 245,0 & 9224 & 2306 & 1025 & 576 & 256 & 144 \\
\hline Total & 257,6 & 10197 & 2549 & 1133 & 637 & 283 & 159 \\
\hline \multicolumn{8}{|c|}{ Ácido ascórbico (mg) } \\
\hline Rural & 54,5 & 456 & 114 & 51 & 29 & 13 & 7 \\
\hline Urbano & 56,4 & 489 & 122 & 54 & 31 & 14 & 8 \\
\hline Total & 55,6 & 475 & 119 & 53 & 30 & 13 & 7 \\
\hline
\end{tabular}

a Con base en valores transformados; (Densidad de nutrientes por 1000 kilocalorías) 0,5.

b Coeficiente (\%) de variación intra-persona (CVw) 
Cada sujeto contesto dos recordatorios del consumo de las últimas 24 horas $(\mathrm{R} 24 \mathrm{H})$ con un intervalo mínimo de una semana para evitar respuestas en base a la memoria. En total se analizaron $1616 \mathrm{R} 24 \mathrm{H}$. El objetivo fue describir las características de la variabilidad de veintisiete variables nutricionales $(\mathrm{Vn})$, establecer las fuentes relativas que aportan a la variabilidad en éstas, determinar el número de días necesarios para acercarse con precisión al verdadero promedio de las $\mathrm{Vn}$ y finalmente, establecer la viabilidad del estudio de las relaciones entre dieta-eventos de salud enfermedad en Colombia. La razón entre varianzas (Sw2/Sb2) fue consistentemente mayor en la zona rural, excepto para la energía y el sodio. El calcio en la zona rural y el cobre en la urbana presentaron la menor razón Sw2/Sb2, 0,36 y 0,12 respectivamente. La vitamina $A$ en la zona rural y el sodio en la urbana, la mayor razón, 0,98 y 0,91 respectivamente. El nivel socioeconómico y el área geográfica fueron los principales determinantes de la variabilidad. Para el consumo de energía, el sexo fue la principal fuente de variación. Para los macronutrientes son necesarios hasta cinco mediciones repetidas para obtener el verdadero promedio de la ingesta de éstos con niveles de error entre 15\% y $40 \%$. Con excepción de la vitamina $A$, todas las razones Sw2/Sb2 se encontraron dentro de valores $<1$, lo que permitiría aún el desarrollo de la epidemiología nutricional en Colombia.

Palabras clave. Dieta; métodos epidemiológicos; análisis de varianza; tamaño de la muestra; Colombia.

\section{BIBLIOGRAFIA}

1. Instituto Colombiano de Bienestar Familiar (ICBF). Encuesta Nacional de la Situación Nutricional en Colombia, 2010. Bogotá, Colombia: ICBF, 2010.

2. Nicole M. Kasper, Herrán OF, Villamor E. Obesity prevalence in Colombian adults is increasing fastest in lower socioeconomic status groups and urban residents: Results from two nationally representative surveys. Public Health Nutr. 2014;2:2398-406.

3. Instituto Nacional de Salud (INS). Observatorio Nacional de Salud. Boletín No. 1. Bogotá: INS. Colombia, 2013.

4. OPS. La salud en las Américas, 2012:92-95. Disponible en: file:///E:/Mis\%20Documentos/Downloads/perfil_col_sa2012.pdf. Consultado en Febrero de 2015.

5. Minsalud. Instituto Nacional de Cancerología (INC). Plan decenal para el control del cáncer en Colombia, 2012-2021. Bogotá: INC. Colombia, 2012.

6. ILSI. Present knowledge in nutrition. 10th edition. John W. Erdman, Jr. (Editor), lan A. MacDonald (Editor), Steven H. Zeisel (Editor). New York: Wiley-BlackWell, 2010.

7. Willet W. Nutritional epidemiology. 3 ed. New York: Oxford University Press, 2012.

8. Margetts BM, Nelson M. Design concepts in nutritional epidemiology. New York: Oxford University Press; 1996.

9. Instituto Nacional de Salud Pública. Encuesta Nacional de Salud y Nutrición 2012 (ENSANUT). Cuernavaca, Morelos: INSP. México, 2012.

10. Ministerio de Salud de la Nación. Encuesta Nacional de Nutrición y Salud 2004-2005. Argentina (ENNyS). Disponible en; www.msal.gov.ar. Consultada en abril de 2014.

11. Office of Nutrition Policy and Promotion Health Products and Food Branch. Canadian Community Health Survey Cycle 2.2, Nutrition (2004). Disponible en: http:// www.hc-sc.gc.ca/fn-an/surveill/nutrition/commun/ cchs_guide_escc-eng.php. Consultada en abril de 2014.

12. CDC. National Health and Nutrition Examination Survey
(NHANES), 2011. Disponible en: http://www.cdc.gov/ nchs/nhanes.htm. Consultada en abril de 2014.

13. Ministerio de Salud. Encuesta Nacional de Indicadores Nutricionales, Bioquímicos, Socioeconómicos y Culturales Relacionados con las Enfermedades Crónico Degenerativas. Perú, 2006. Disponible en: http://www. minsa.gob.pe. Consultada en abril de 2014.

14. Ministerio da Saúde. Pesquisa nacional de demografia e saúde da crianca e da mulher. Brasilia/DF, 2008. Disponible en: http://bvsms.saude.gov.br/bvs/pnds/index. php. Consultada en abril de 2014.

15. Popkin BM, Gordon-Larsen P. The nutrition transition: worldwide obesity dynamics and their determinants. Int J Obes. 2004;28:S2-S9.

16. Contreras J. Alimentación y religión. Humanitas Humanidades Médicas. 2007;16:1-22. Disponible en: http://www. fundacionmhm.org/www_humanitas_es_numero16/ articulo.pdf. Consultado en agosto de 2014.

17. Monteiro CA. Nutrition and health. The issue is not food, nor nutrients, so much as processing. Public. Health. Nutr. 2009;12; 729-31.

18. Monteiro CA, Levy RB, Claro RM, Ribeiro IR, Cannon G. Increasing consumption of ultra-processed foods and likely impact on human health: evidence from Brazil. Public Health Nutr. 2010; 14:5-13.

19. Ocampo PR, Prada GE, Herrán OF. Patrones de consumo alimentario y exceso de peso infantil; Encuesta de la Situación Nutricional en Colombia, 2010. Rev Chil Nutr. 2014:41:351-59.

20. Jahns L, Siega-Riz A, Popkin B. The increasing prevalence of snacking among US children from 1977 to 1996. J Pediatr. 2001; 138: 493-8.

21. Beaton GH, Milner J, Corey P, McGuire S, Cousins M, Stewart E, et al. Sources of variance in 24-hour dietary recall data: implications for nutrition study design and interpretation. Am J Clin Nutr. 1979;32:2546-59.

22. Herrán OF, Quintero DC, Ardila MF. Sources and magnitude of the variation in the diet of Bucaramanga, Colombia. Rev Chil Nutr. 2006;33:55-64.

23. Nelson M, Black AE, Morris JA, Cole TJ. Between- and within-subject variation in nutrient intake from infancy to old age: Estimating the number of days required to rank dietary intakes with desired precision. Am. J. Clin. Nutr. 1989;50:155-67.

24. Monterrey P, Cortés Y, Corredor C, Acosta A, Caicedo P. Variabilidad de la dieta de mujeres jóvenes universitarias, su influencia sobre los criterios de evaluación dietética. Perspect Nutr Hum. 2009;11 :11-24.

25. Tokudome $Y$, Imaeda $N$, Nagaya $T$, Ikeda $M$, Fujiwara $N$, Sato J, et al. Daily, weekly, seasonal, within and between individual variation in nutrient intake according to four season consecutive 7 day weighed diet records in Japanese female dietitians. J Epidemiol. 2002;12:85-92.

26. Palaniappan U, Cue RI, Payette H, Gray-Donald K. Implications of day-to-day variability on measurements of usual food and nutrient intakes. J Nutr. 2003;133: 232-5.

27. Nyambose J, Kosky KG, Tucker KL. High intra/interindividual variance ratios for energy and nutrient intakes of pregnant women in rural Malawi show that many days are required to estimate usual intake. J Nutr. 2002; 132:1313-8.

28. Heredia P, Del Castillo S, Fonseca Z, Chacón O, Herrán OF. Base de datos de la composición nutricional de alimentos y preparaciones: Compilación de las universidades Nacional de Colombia y Universidad Industrial 
de Santander. Bogotá, Colombia, 2009.

29. Universidad Nacional de Colombia. Departamento de Nutrición. Proceso de estandarización de pesos de modelos para la estimación del tamaño de ración. Bogotá, Colombia; 2009. (Informe Técnico)

30. Ardila MF, Chacón OA, Herrán OF. Consumo dietario y estado de nutrición en población colombiana. Rev Chil Nutr. 2014;41:8-16.

31. Jiménez AZ, Prada GE, Herrán OF. Escalas para medir la seguridad alimentaria en Colombia. ¿Son válidas? Rev Chil Nutr. 2012;39:8-17.

32. Camargo MI, Quintero DC, Herrán OF. Seguridad alimentaria en Colombia y modelo Rasch. Rev Chil Nutr. 2012;39:168-80.

33. Departamento Nacional de Planeación (DPN). Sistema de Identificación de Potenciales Beneficiarios de Programas Sociales (SISBEN). Disponible en; https://www. sisben.gov.co/EISisb\%C3\%A9n.aspx\#.VNKDP52G-So. Consultado en diciembre de 2014.

34. Carriquiry AL. Estimation of usual intake distributions of nutrients and foods. J Nutr. 2003;133:601s-8s.

35. Iowa State University. PC-Side, versión 1,02. lowa: Department of Statistics and Center for Agricultural and Rural Development; 2004.

36. Fritsch HM, Salgado-Martínez H (Editores). Manual de encuestas de dieta. Perspectivas en salud pública, No.
23. Cuernavaca, México. Instituto Nacional de Salud Pública, 1996. p. 25-50.

37. Stata Statistical Software: Release 13.1. Collegue Station, TX: StataCorp LP.

38. McDonald CM, Baylin A, Arsenault JE, Mora-Plazas M, Villamor E. Overweight is more prevalent than stunting and is associated with socioeconomic status, maternal obesity, and a snacking dietary pattern in school children from Bogotá, Colombia. J Nutr. 2009;139:370-6.

39. PNUD. Colombia rural: Razones para la esperanza. Informe nacional de desarrollo humano, 2011. Bogotá: PNUD, 2011.

40. Erkkola M, Kyttala P, Takkinen HM, Kronberg-Kippila C, Nevalainen J, Simell O, et al. Nutrient intake variability and number of days needed to assess intake in preschool children. Brit J Nutr. 2011;106:130-40.

41. Basiotis P, Welsh S, Cronin F Kelsay J Mertz W. Number of days of food intake records required to estimate individual and groupnutrient intakes with defined confidence. J Nutr. 1987;117:1638-41.

42. DelCastillo S. La situación nutricional de la niñez en Latinoamérica: entre la deficiencia y el exceso, de brecha nutricional a deuda social. Biomédica. 2012;32: (Editorial).

43. MinSalud, FAO, OSAN. Aproximación a los determinantes de la doble carga nutricional en Colombia. Boletín No. 004/2014. Bogotá: MinSalud. Colombia, 2014. 